(MacWilliams), Hydra (Bode and Bode), and in more complex organisms such as insects (Sander; Anderson and NüssleinVolhard), higher plants (Sachs, McDaniel), sharks (Reif), amphibians (Malacinski, Slack, Cooke, Stocum and Fallon) and chicks (Dhouailly), force an elaboration of concepts beyond either the original positional information theory or the polar coordinate model. Here we find a profusion of developmental mechanisms, some classical and some modern, all very. clearly elaborated. Both Kauffman and Meinhardt achieve some success in accounting for certain aspects of these phenomena by the discovery of new properties of old models, and by adding dynamical features with switching logic and recursive properties to generate structures such as segments and compartments, and models of distal transformation in insects.

But for me the high point of the volume, whence one catches a glimpse of a way of putting together all that has come before but within a somewhat different scheme, arose from a certain reading of the chapters by Karlsson, Poethig and Nuccitelli - the first on Drosophila, the second on angiosperm leaf morphogenesis and the third on electrical fields in morphogenesis (plant and animal, unicellular and multicellular). The first two authors interpret pattern formation in terms of fields of mechanical force (mediated by the cytoskeleton) that both guide and are guided by patterns of cell division and differentiation in Drosophila imaginal discs and in leaf meristems; while the Nuccitelli paper on ionic currents indicates the dynamic boundary conditions for affecting the state of the cytoskeleton and cell metabolism.

Neither Karlsson nor Poethig sees evidence in their material for either a logical gene-regulated decision process resulting in spatial patterns, or for gradients or "coordinate-systems" that spatially control such decisions. Since Sydney Brenner, somewhat impishly, has recently said about the explanation of pattern formation by molecular mechanisms of gene control: "I doubt whether anyone believes that anymore", we can now feel free to seek alternatives. One possibility is to understand morphogenesis as a unitary process in which global mechanical fields (of strain, or strain-rate, for example), acting through and on both the cytoskeleton and cell membranes, result in ion fluxes and associated elastic and plastic deformations of tissue that themselves generate further stresses, ion flows and other biochemical changes (including, of course, gene activities), with reciprocal interactions. After an extended exploration of the information and programming metaphors, the subject may be returning to the themes of Entwicklungsmechanik; which would have pleased Driesch.

Brian Goodwin is Professor of Biology at the Open University.

\section{Trains of thought}

John C. Marshall

Vehicles: Experiments in Synthetic

Psychology.

By Valentino Braitenberg.

MIT Press: 1984. Pp. 152.

$\$ 14.95, £ 15.60$.

William Thomson, Lord Kelvin, is reputed to have remarked: "If you can make a mechanical model of it you understand it, and if you can't you don't". Kelvin was, of course, talking about the role of models in physics (and thinking about the success of his tidal wave predictor), but he could equally well have had psychological models in mind.

There is a tradition in theoretical psychology, extending back some two millennia, whereby the explanation of behaviour is explicitly equated with the construction of "toys" that exhibit the behaviour in question. Some of the outstanding contributors to this enterprise include Heron of Alexandria, who built a complex automaton wherein, on the lifting of a golden apple, a miniature Hercules shot a snake that then hissed; Albertus Magnus, whose magnum opus, a life-sized automatic servant, was destroyed, so legend goes, by Thomas Aquinas who suspected it to be the Devil's work; Jacques de Vaucanson, the constructor of an eating, drinking and quacking artificial duck, and of a flute-player that out-performed many human virtuosi; and the Jaquet-Droz (père et fils), whose dolls could write and draw with amazing facility. Like Magnus, the elder Droz and his models suffered a fate more extreme than failure to obtain tenure. Following a public exhibition of the writing-boy, Hermann von Helmholtz reminds us that "... this boy and its maker, being suspected of the black art, lay for a time in the Spanish Inquisition, and with difficulty obtained their freedom'".

Closer to our own time, Grey Walter and Claude Shannon devised, respectively, tortoises and mice that ran mazes in electronic instantiation of the laws of classical learning-theory. To this distinguished line of artificial intelligentsia we must now add Valentino Braitenberg, a director of the Max Planck Institute of Biological Cybernetics at Tübingen.

Professor Braitenberg's Vehicles is precisely that; a series of 14 little vehicles, with surprisingly life-like properties. The sequence begins with a simple bug where one sensor is connected to one motor such that "the more there is of the quality to which the sensor is tuned, the faster the motor goes" and ends with creatures of more Rococo wiring that appear to demonstrate foresight, egotism and optimism. Each successive creature embodies the critical structures of all previous models and adds one further feature; the vehicles thereby illustrate a single line of evolu- tionary ascent and painlessly introduce the unsuspecting reader to the basic concepts of self-regulating machines. Throughout, Professor Braitenberg writes in a dry, tongue-in-cheek fashion, poking sly fun at any philosopher (or Reith lecturer) whose hand is too unsteady to wield Occam's razor. He is thus a worthy successor to Julien Offray de la Mettrie, who in his L'Homme Machine of 1748, argued that

... since all the faculties of the soul depend to such a degree on the proper organization of the brain and of the whole body, that apparently they are but this organization itself, the soul is clearly an enlightened machine.

For both la Mettrie and Braitenberg, it's Chinese rooms all the way down.

In the main text, Professor Braitenberg's humour serves to illuminate his serious moral, "the law of uphill analysis and downhill invention", that is:

It is pleasurable and easy to create little machines that do certain tricks. It is also quite easy to observe the full repertoire of behavior of these machines - even if it goes beyond what we had originally planned, as it often does. But it is much more difficult to start from the outside and to try to guess internal structure just from the observation of behavior.

Worse,

it is actually impossible in theory to determine exactly what the hidden mechanism is without opening the box, since there are always many different mechanisms with identical behavior.

Accordingly, Professor Braitenberg provides an extensive appendix of biological notes on his vehicles. Here he sketches the analogies between his creatures and those of the given, natural world, leading one lightly into the complexities of current neuroanatomy and neurophysiology. The transition between the stark formalism of the hardware vehicles and the cell-synapse-circuit wetware that they mimic is mediated by a portfolio of phantasmagoric drawings by Maciek Albrecht.

For both amusement and enlightenment, Vehicles can be unhesitatingly recommended to anyone (student or teacher) involved in courses on "Brain and Behaviour"'. The volume would also make a marvellous Christmas present for any intelligent teenager interested in either computing or animal-watching, or better, both.

John C. Marshall is in the Neuropsychology Unit, part of the Neuroscience Group at the Radcliffe Infirmary, Oxford.

\section{New in paperback}

- The Structure of Time by W. H. NewtonSmith. Publisher is Routledge \& Kegan Paul, price is $£ 7.95, \$ 14.95$.

- Curious Naturalists by Niko Tinbergen, a reprint of the revised (1974) edition of the book. Publisher is The University of Massachusetts Press, price is $\$ 9.95$.

- Plant Biotechnology, edited by S. H. Mantell and H. Smith, Vol. 18 in the seminar series of the Society for Experimental Biology. Publisher is Cambridge University Press, price is $£ 12.50$, $\$ 19.95$. 